\title{
Combinações de Variáveis Aleatórias Beta Tipo II Aplicadas em Modelagem Hidrológica
}

\section{Combinations of Beta Type II Random Variables Applied in Hydrologic Modeling}

\author{
Jailson de Araujo Rodrigues \\ Departamento de Ciências Exatas \\ Universidade Federal de Lavras - UFLA, Lavras, MG \\ jailsondearanjo@yahoo.com.br \\ Ana Paula Coelho Madeira Silva \\ Universidade Federal de São João del-Rei - UFSJ, Sete Lagoas, MG \\ anapaula@ufsj.edu.br \\ Lucas Monteiro Chaves \\ Departamento de Ciências Exatas \\ Universidade Federal de Lavras - UFLA, Lavras, MG \\ lucas@dex.ufla.br \\ Fredy Walther Castellares Cáceres \\ Instituto de Ciências Exatas \\ Universidade Federal de Minas Gerais - UFMG, Belo Horizonte, MG \\ fredy@est.ufmg.br
}

Resumo: As distribuições do produto e do quociente de variáveis aleatórias são utilizadas em diversas áreas das ciências aplicadas e têm sido intensivamente estudadas por vários pesquisadores. Nesse trabalho, são deduzidas as distribuições exatas de $X Y, X / Y$ e $X /(X+Y)$ quando $X$ e $Y$ são variáveis beta tipo II e independentes. Os resultados obtidos são aplicados na análise de dados reais de precipitações pluviométricas ocorridas em cidades do Estado de Sergipe.

Palavras-chave: combinações de variáveis aleatórias; distribuição beta tipo II; mode-

Recebido em 28/02/2012 - Aceito em 24/06/2012.

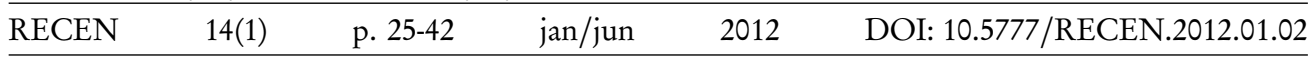


lagem hidrológica.

\begin{abstract}
The distributions of products and ratios of random variables are used in various fields of applied science and they have been intensively studied by several researchers. In this work they are deducted the exact distributions of $\mathrm{X} Y, X / Y$ and $\mathrm{X} /(\mathrm{X}+\mathrm{Y})$ when $\mathrm{X}$ and $\mathrm{Y}$ are independent beta type II random variables. The results found are applied to real data analysis of rainfall occurred in cities in the state of Sergipe.
\end{abstract}

Key words: beta type II distribution; combinations of random variables; hydrological modeling.

\title{
1 Introdução
}

As distribuições de combinações de variáveis surgem naturalmente em problemas provenientes de diversas áreas do conhecimento. Particularmente, combinações do tipo $X Y, X / Y$ e $X /(X+Y)$, quando $X$ e $Y$ são independentes e pertencem à mesma família de distribuições, têm sido intensivamente estudadas por vários pesquisadores. Tratando-se do produto de variáveis da mesma família, tem-se: Sakamoto [1] para a distribuição uniforme; Podolski, Springer, Thompson e Stuart [2-4] com relação à família gama; Harter e Wallgren [5, 6] para a distribuição t de Student; Bhargava, Khatri, Steece, Tang e Gupta [7-9] abordando a família beta; Malik e Trudel [10] para o modelo exponencial; Nadarajah e Gupta [11] para a família Bessel; Nadarajah e Ali [12] com relação à distribuição Pareto; e Ali, Pal e Woo [13] para o modelo Pareto exponenciado. Tratando-se do quociente de variáveis oriundas da mesma família, ver Korhonen, Narula e Marsaglia [14,15] para a família normal; Provost [16] para o modelo t de Student; Basu e Lochner [17] com relação à distribuição Weibull; Prudnikov et al [18] para variáveis gama; Podolski [19] abordando a família beta; Nadarajah e Gupta [11] para as variáveis Bessel e, com respeito à distribuição Pareto, o problema foi estudado por Nadarajah e Ali [12].

Alguns pesquisadores também estão trabalhando em combinações de variáveis 
quando $X$ e $Y$ provêm de famílias diferentes de distribuições. Por exemplo, o produto e o quociente de variáveis aleatórias $t$ de Student e logística foram estudados por Nadarajah e Ali [20], o mesmo foi feito por Nadarajah e Kotz [21] para variáveis gama e beta. Nadarajah [22] determinou o produto e o quociente de variáveis Laplace e Bessel. Nadarajah e Kotz estabeleceram as distribuições do produto e do quociente de variáveis Pearson tipo VII e Laplace [23], variáveis gama e Weibull [24], variáveis aleatórias t de Student e Bessel [25] e distribuição do quociente de uma variável gama com uma variável Levy [26]. Shakil et al [27] estabeleceram o quociente de uma variável gama com uma variável Rayleigh; e o quociente de uma variável Maxwell com uma variável Rayleigh foi estudado por Springer e Thompson [28]. Recentemente, a dedução das distribuições da combinação linear, produto e quociente de variáveis aleatórias normal e Laplace foram apresentadas por Nadarajah e Kotz [29].

Neste trabalho, é apresentado um estudo das distribuições de $X Y, X / Y$ e $X /(X+$ $Y)$ quando $X$ e $Y$ são variáveis aleatórias independentes beta tipo II distribuídas. $\mathrm{Na}$ seção 2, é apresentado o modelo beta tipo II, sua relação com o modelo beta tipo I e algumas funções especiais envolvidas nos cálculos das distribuições. Na seção 3, são deduzidas as funções densidade de probabilidade das variáveis $X Y, X / Y$ e $X /(X+Y)$. Finalmente, na seção 4, os resultados obtidos são aplicados na análise de precipitações pluviométricas ocorridas em cidades do Estado de Sergipe.

\section{Distribuições beta}

Distribuição beta tipo I: Uma variável aleatória $X$ tem distribuição beta tipo I com parâmetros $\alpha>0$ e $\beta>0$ quando sua função densidade de probabilidade (fdp) é da forma:

$$
f(x)=\frac{x^{\alpha-1}(1-x)^{\beta-1}}{B(\alpha, \beta)}
$$

em que $0<x<1$ e $B(\alpha, \beta)$ representa a função beta,

$$
B(\alpha, \beta)=\int_{0}^{1} t^{\alpha-1}(1-t)^{\beta-1} d t
$$

Simbolicamente, quando $X$ tem distribuição dada por (1), escreve-se $X \sim B I(\alpha, \beta)$. 
Distribuição beta tipo II: Uma variável aleatória $X$ tem distribuição beta tipo II com parâmetros $\alpha>0$ e $\beta>0$ quando sua função densidade de probabilidade (fdp) é da forma:

$$
f(x)=\frac{x^{\alpha-1}(1+x)^{-(\alpha+\beta)}}{B(\alpha, \beta)}
$$

em que $0<x$. Neste caso, escreve-se simbolicamente $X \sim B I I(\alpha, \beta)$.

As duas distribuições beta estão relacionadas intrinsecamente por meio de uma transformação. Se $X \sim B I(\alpha, \beta)$, então $Z=(1-X) / X \sim B I I(\beta, \alpha)$. Reciprocamente, se $Z \sim B I I(\beta, \alpha)$, então $X=1 /(1+Z) \sim B I(\alpha, \beta)$. Várias características referentes aos dois modelos, inclusive a propriedade mencionada, podem ser encontradas em [30].

Além da função beta, os cálculos envolvidos no trabalho requerem o uso de outras funções especiais como a função gama,

$$
\Gamma(\alpha)=\int_{0}^{\infty} t^{\alpha-1} \exp (-t) d t
$$

e a função hipergeométrica de Gauss,

$$
{ }_{2} F_{1}(a, b ; c ; x)=\sum_{k=0}^{\infty} \frac{(a)_{k}(b)_{k} x^{k}}{(c)_{k} k !}
$$

em que $(d)_{k}=d(d+1) \ldots(d+k-1)$ denota o fatorial ascendente, $k \in \mathbb{Z}_{+}$. As propriedades dessas funções especiais podem ser vistas em [31].

\section{Funções densidade de probabilidade}

Nos Teoremas de 1 a 3 são deduzidas as fdp de $P=X Y, R=X / Y$ e $Q=X /(X+$ $Y)$ quando $X$ e $Y$ são beta tipo II distribuídas e independentes.

Lema 1 (Equação (2.2.6.24), [32]]). Se $0<y, 0<z e 0<\alpha<\rho+\gamma$, então,

$$
\int_{0}^{\infty} x^{\alpha-1}(x+y)^{-\rho}(x+z)^{-\gamma} d x=z^{-\gamma} y^{\alpha-\rho} B(\alpha, \rho+\gamma-\alpha){ }_{2} F_{1}\left(\alpha, \gamma ; \rho+\gamma ; 1-\frac{y}{z}\right)
$$


Teorema 1 Se $X \sim B I I(\alpha, \beta)$ e $Y \sim B I I(\theta, \lambda)$ são independentes, então, a fdp de $P=X Y$ pode ser escrita como:

$$
f_{P}(p)=\frac{B(\alpha+\lambda, \beta+\theta) p^{-(\lambda+1)}}{B(\alpha, \beta) B(\theta, \lambda)}{ }_{2} F_{1}\left(\alpha+\lambda, \theta+\lambda ; \alpha+\beta+\theta+\lambda ; 1-\frac{1}{p}\right)
$$

sendo que $0<p$.

Prova: Utilizando a mudança de variáveis $(X, P)=(X, X Y)$ o Jacobiano dessa transformação é dado por

$$
J=\left|\begin{array}{ll}
\frac{\partial x}{\partial x} & \frac{\partial x}{\partial y} \\
\frac{\partial p}{\partial x} & \frac{\partial p}{\partial y}
\end{array}\right|=\left|\begin{array}{cc}
1 & 0 \\
y & x
\end{array}\right|=x
$$

Assim, a fdp conjunta de $(X, P)$ é dada por

$$
\begin{aligned}
f_{X, P}(x, p) & =f_{X, Y}\left(x, \frac{p}{x}\right) \times|J|^{-1} \\
& =\frac{p^{\theta-1} x^{\alpha+\lambda-1}(1+x)^{-(\alpha+\beta)}(x+p)^{-(\theta+\lambda)}}{B(\alpha, \beta) B(\theta, \lambda)}
\end{aligned}
$$

Pode-se, então, escrever a fdp da variável $P$ da seguinte maneira:

$$
\begin{aligned}
f_{P}(p) & =\int_{0}^{\infty} f_{X, P}(x, p) d x \\
& =\frac{p^{\theta-1}}{B(\alpha, \beta) B(\theta, \lambda)} \int_{0}^{\infty} x^{\alpha+\lambda-1}(1+x)^{-(\alpha+\beta)}(x+p)^{-(\theta+\lambda)} d x
\end{aligned}
$$

A conclusão do teorema decorre da aplicação do Lema 1 em (9).

Teorema 2 Se $X \sim B I I(\alpha, \beta)$ e $Y \sim B I I(\theta, \lambda)$ são independentes, então, a fdp de $R=X / Y$ pode ser escrita como:

$$
f_{R}(r)=\frac{B(\alpha+\theta, \beta+\lambda) r^{-(\theta+1)}}{B(\alpha, \beta) B(\theta, \lambda)}{ }_{2} F_{1}\left(\alpha+\theta, \theta+\lambda ; \alpha+\beta+\theta+\lambda ; 1-\frac{1}{r}\right)
$$


Revista Ciências Exatas e Naturais, Vol.14, n 1, Jan/Jun 2012

sendo que $0<r$.

Prova: Utilizando a mudança de variáveis $(X, R)=(X, X / Y)$ o Jacobiano dessa transformação é dado por

$$
J=\left|\begin{array}{ll}
\frac{\partial x}{\partial x} & \frac{\partial x}{\partial y} \\
\frac{\partial r}{\partial x} & \frac{\partial r}{\partial y}
\end{array}\right|=\left|\begin{array}{cc}
1 & 0 \\
\frac{1}{y} & -\frac{x}{y^{2}}
\end{array}\right|=-\frac{x}{y^{2}}
$$

Assim, a fdp conjunta de $(X, R)=(X, X / Y)$ é dada por

$$
\begin{aligned}
f_{X, R}(x, r) & =f_{X, Y}\left(x, \frac{x}{r}\right) \times|J|^{-1} \\
& =\frac{r^{\lambda-1} x^{\alpha+\theta-1}(1+x)^{-(\alpha+\beta)}(x+r)^{-(\theta+\lambda)}}{B(\alpha, \beta) B(\theta, \lambda)}
\end{aligned}
$$

Pode-se, então, escrever a fdp da variável $R$ da seguinte maneira:

$$
\begin{aligned}
f_{R}(r) & =\int_{0}^{\infty} f_{X, R}(x, r) d x \\
& =\frac{r^{\lambda-1}}{B(\alpha, \beta) B(\theta, \lambda)} \int_{0}^{\infty} x^{\alpha+\theta-1}(1+x)^{-(\alpha+\beta)}(x+p)^{-(\theta+\lambda)} d x
\end{aligned}
$$

A conclusão do teorema decorre da aplicação do Lema 1 em (13).

Teorema 3 Se $X \sim B I I(\alpha, \beta)$ e $Y \sim B I I(\theta, \lambda)$ são independentes, então, a fdp de $Q=X /(X+Y)$ pode ser escrita como:

$$
f_{Q}(q)=\frac{B(\alpha+\theta, \beta+\lambda)}{B(\alpha, \beta) B(\theta, \lambda)} q^{-(\theta+1)}(1-q)^{\theta-1}{ }_{2} F_{1}\left(\alpha+\theta, \lambda+\theta ; \alpha+\beta+\theta+\lambda ; 2-\frac{1}{q}\right)
$$

sendo que $0<q<1$. 
Prova: Utilizando a mudança de variáveis $(U, Q)=(X+Y, X /(X+Y))$ o Jacobiano dessa transformação é dado por

$$
J=\left|\begin{array}{ll}
\frac{\partial u}{\partial x} & \frac{\partial u}{\partial y} \\
\frac{\partial q}{\partial x} & \frac{\partial q}{\partial y}
\end{array}\right|=\left|\begin{array}{cc}
1 & 1 \\
\frac{y}{(x+y)^{2}} & -\frac{x}{(x+y)^{2}}
\end{array}\right|=-\frac{1}{x+y}
$$

Assim, a fdp conjunta de $(U, Q)=(X+Y, X /(X+Y))$ é dada por

$$
\begin{aligned}
f_{U, Q}(u, q) & =f_{X, Y}(u q, u(1-q)) \times|J|^{-1} \\
& =\frac{(u q)^{\alpha-1}(1+u q)^{-(\alpha+\beta)}[u(1-q)]^{\theta-1}[1+u(1-q)]^{-(\theta+\lambda)} u}{B(\alpha, \beta) B(\theta, \lambda)} \\
& =\frac{u^{\alpha+\theta-1} q^{\alpha-1}(1-q)^{\theta-1}(1+u q)^{-(\alpha+\beta)}[1+(1-q) u]^{-(\theta+\lambda)}}{B(\alpha, \beta) B(\theta, \lambda)}
\end{aligned}
$$

Pode-se então, escrever a fdp da variável $Q$ da seguinte maneira:

$$
\begin{aligned}
f_{Q}(q) & =\int_{0}^{\infty} f_{U, Q}(u, q) d u \\
& =\frac{q^{\alpha-1}(1-q)^{\theta-1}}{B(\alpha, \beta) B(\theta, \lambda)} \int_{0}^{\infty} u^{\alpha+\theta-1}(1+u q)^{-(\alpha+\beta)}[1+(1-q) u]^{-(\theta+\lambda)} d u
\end{aligned}
$$

reescrevendo (17) de forma conveniente, tem-se

$$
f_{Q}(q)=\frac{q^{-(\beta+1)}(1-q)^{-(\lambda+1)}}{B(\alpha, \beta) B(\theta, \lambda)} \int_{0}^{\infty} u^{\alpha+\theta-1}\left(\frac{1}{q}+u\right)^{-(\alpha+\beta)}\left(\frac{1}{1-q}+u\right)^{-(\theta+\lambda)} d u
$$

Aplicando o Lema 1 em 18$]$ chega-se a conclusão do teorema.

Nas figuras de 1 a 3 são ilustradas as formas das fdp $P=X Y, R=X / Y$ e $Q=$ $X /(X+Y)$ para diferentes valores paramétricos. 
Revista Ciências Exatas e Naturais, Vol.14, n 1, Jan/Jun 2012

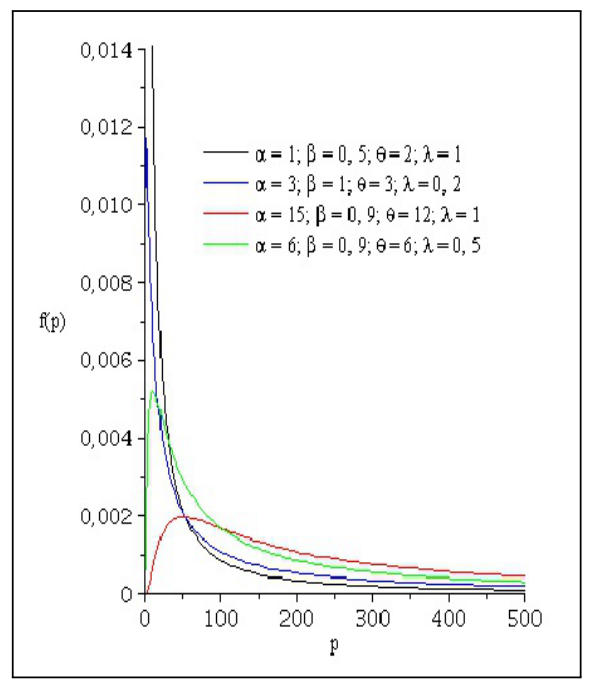

Figura 1. Gráficos da fdp de $P=X Y$ para diferentes valores paramétricos.

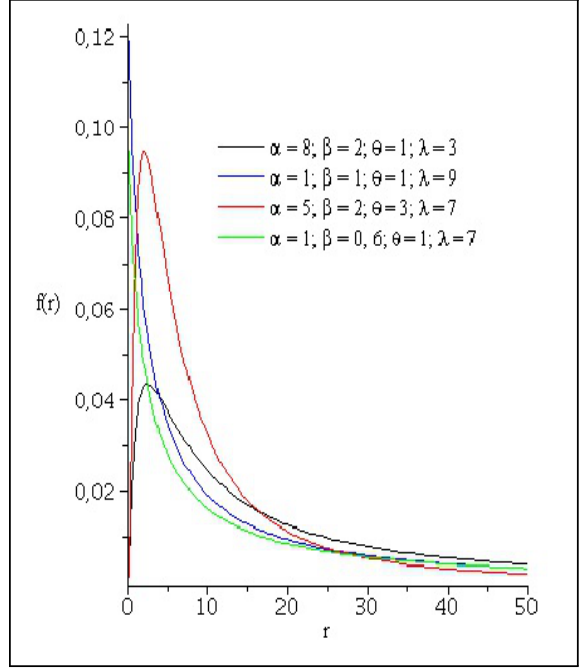

Figura 2. Gráficos da fdp de $R=X / Y$ para diferentes valores paramétricos.

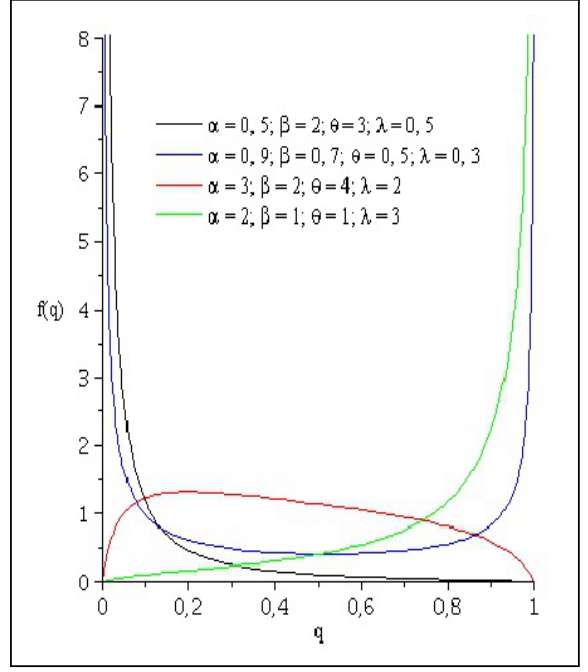

Figura 3. Gráficos da fdp de $Q=X /(X+Y)$ para diferentes valores paramétricos.

\section{Aplicação}

Nesta seção, é feita uma aplicação do modelo na análise de dados de precipitações pluviométricas ocorridas nas cidades de Aquidabã, Aracaju, Boquim, Nossa Senhora 
da Glória, Itabaiana, Porto da Folha, Poço Redondo e Umbaúba, todas situadas no Estado de Sergipe.

Os dados explorados foram coletados em estações agrometeorológicas e hidrometeorológicas do Centro de Meteorologia da Secretaria de Estado do Meio Ambiente e dos Recursos Hídricos do Estado de Sergipe (SEMARH); as coordenadas geográficas e os tipos das estações são exibidos na tabela 1. O conjunto de dados pode ser acessado no seguinte endereço eletrônico: http://www.semarh.se.gov.br/meteorologia/ modules/tinydo/index.php?id $=50$. A base de dados corresponde às medições diárias do índice pluviométrico no período de 01 de janeiro de 2009 até 31 de dezembro de 2010.

Tabela 1. Localização e tipo das estações

\begin{tabular}{cllll}
\hline Cidades & Latitude & Longitude & Altitude & Tipo \\
\hline Aquidabã & $-10,29^{\circ}$ & $-37,02^{\circ}$ & $218 \mathrm{~m}$ & Hidrometeorológica \\
Aracaju & $-10,95^{\circ}$ & $-37,05^{\circ}$ & $4 \mathrm{~m}$ & Hidrometeorológica \\
Boquim & $-11,14^{\circ}$ & $-37,62^{\circ}$ & $160 \mathrm{~m}$ & Hidrometeorológica \\
Itabaiana & $-10,70^{\circ}$ & $-37,42^{\circ}$ & $200 \mathrm{~m}$ & Agrometeorológica \\
N. S. da Glória & $-10,20^{\circ}$ & $-37,43^{\circ}$ & $300 \mathrm{~m}$ & Agrometeorológica \\
Porto da Folha & $-10,00^{\circ}$ & $-37,42^{\circ}$ & $149 \mathrm{~m}$ & Hidrometeorológica \\
Poço Redondo & $-9,84^{\circ}$ & $-37,67^{\circ}$ & $260 \mathrm{~m}$ & Agrometeorológica \\
Umbaúba & $-11,38^{\circ}$ & $-37,67^{\circ}$ & $153 \mathrm{~m}$ & Hidrometeorológica \\
\hline
\end{tabular}

Utilizando as medições do índice pluviométrico, obtêm-se os dados sobre período de dias com ocorrência de precipitações $(X)$ e período contíguo de dias sem ocorrência de precipitação $(Y)$. O objetivo é modelar a proporção de dias com ocorrência de precipitações, o que pode ser feito pela distribuição de $Q=X /(X+Y)$. Nesse contexto, assume-se que $X$ e $Y$ são beta tipo II distribuídas e independentes. A validade da suposição de independência foi verificada por meio do teste $\rho$ de Spearman [33]. Os p-valores obtidos no teste foram acima de 0,05 (Tabela 2), o que confirma a hipótese de independência entre as amostras observadas de $X$ e $Y$ para as oito cidades estudadas. 
Revista Ciências Exatas e Naturais, Vol.14, n 1, Jan/Jun 2012

\begin{tabular}{cc} 
Tabela & \multicolumn{2}{l}{ 2. p-valor do teste $\rho$ de Sperm } \\
\cline { 2 - 2 } & \multicolumn{2}{l}{ Cidades } & p-valor \\
\hline Aquidabã & 0,061 \\
Aracaju & 0,239 \\
Boquim & 0,541 \\
Itabaiana & 0,411 \\
N. S. da Glória & 0,795 \\
Porto da Folha & 0,142 \\
Poço Redondo & 0,692 \\
Umbaúba & 0,150 \\
\hline
\end{tabular}

O ajuste das distribuições foi feito via Método da Máxima Verossimilhança. Se $\left(X_{1}, Y_{1}\right), \ldots,\left(X_{n}, Y_{n}\right)$ representa uma amostra aleatória conjunta de $X \sim B I I(\alpha, \beta)$ e $Y \sim B I I(\theta, \lambda)$, então, as estimativas de máxima verossimilhança de $\alpha$ e $\beta$ são as soluções do sistema de equações não-lineares:

$$
\begin{gathered}
n \psi(\widehat{\alpha}+\widehat{\beta})-n \psi(\widehat{\alpha})=\sum_{i=1}^{n} \log \left(1+x_{i}\right)-\sum_{i=1}^{n} \log \left(x_{i}\right) \\
n \psi(\widehat{\alpha}+\widehat{\beta})-n \psi(\widehat{\beta})=\sum_{i=1}^{n} \log \left(1+x_{i}\right)
\end{gathered}
$$

Analogamente, as estimativas de máxima verossimilhança de $\theta$ e $\lambda$ são as soluções do sistema de equações:

$$
\begin{gathered}
n \psi(\widehat{\theta}+\widehat{\lambda})-n \psi(\widehat{\theta})=\sum_{i=1}^{n} \log \left(1+y_{i}\right)-\sum_{i=1}^{n} \log \left(y_{i}\right) \\
n \psi(\hat{\theta}+\widehat{\lambda})-n \psi(\widehat{\lambda})=\sum_{i=1}^{n} \log \left(1+y_{i}\right)
\end{gathered}
$$

em que $\psi(\cdot)$ denota função digama $\psi(t)=d \log \Gamma(t) / d t$. Essas equações não possuem solução fechada, sendo necessária uma implementação numérica para resolvê-las, o que foi feito utilizando um algoritmo quasi-Newton função $\mathrm{nlm}$ do pacote stats no ambiente computacional $\mathrm{R}$ [34]. As matrizes de informação associadas são respecti- 
vamente,

$$
\left[\begin{array}{cc}
n\left[\psi^{\prime}(\alpha+\beta)-\psi^{\prime}(\alpha)\right] & n \psi^{\prime}(\alpha+\beta) \\
n \psi^{\prime}(\alpha+\beta) & n\left[\psi^{\prime}(\alpha+\beta)-\psi^{\prime}(\beta)\right]
\end{array}\right]
$$

e

$$
\left[\begin{array}{cc}
n\left[\psi^{\prime}(\theta+\lambda)-\psi^{\prime}(\theta)\right] & n \psi^{\prime}(\theta+\lambda) \\
n \psi^{\prime}(\theta+\lambda) & n\left[\psi^{\prime}(\theta+\lambda)-\psi^{\prime}(\lambda)\right]
\end{array}\right]
$$

Invertendo a matriz de informação $[19)$, obtém-se a variância e covariância dos estimadores de máxima verossimilhança de $\alpha$ e $\beta$,

$$
\begin{gathered}
\operatorname{Var}(\widehat{\alpha})=\frac{\psi^{\prime}(\alpha+\beta)-\psi^{\prime}(\beta)}{n\left\{\psi^{\prime}(\alpha) \psi^{\prime}(\beta)-\psi^{\prime}(\alpha+\beta)\left[\psi^{\prime}(\alpha)+\psi^{\prime}(\beta)\right]\right\}} \\
\operatorname{Var}(\widehat{\beta})=\frac{\psi^{\prime}(\alpha+\beta)-\psi^{\prime}(\alpha)}{n\left\{\psi^{\prime}(\alpha) \psi^{\prime}(\beta)-\psi^{\prime}(\alpha+\beta)\left[\psi^{\prime}(\alpha)+\psi^{\prime}(\beta)\right]\right\}} \\
\operatorname{Cov}(\widehat{\alpha}, \widehat{\beta})=-\frac{\psi^{\prime}(\alpha+\beta)}{n\left\{\psi^{\prime}(\alpha) \psi^{\prime}(\beta)-\psi^{\prime}(\alpha+\beta)\left[\psi^{\prime}(\alpha)+\psi^{\prime}(\beta)\right]\right\}}
\end{gathered}
$$

A variância e covariância dos estimadores de máxima verossimilhança de $\theta$ e $\lambda$ podem ser calculadas utilizando fórmulas análogas as expressões 21), 22] e 23), respectivamente. Na tabela 3 , são exibidas as estimativas obtidas para os parâmetros $\alpha$, $\beta, \theta$ e $\lambda$, juntamente com os respectivos erros padrões.

Tabela 3. Estimativas dos parâmetros e erros padrões

\begin{tabular}{lllll}
\hline Cidades & $\hat{\alpha}(\mathrm{ep})$ & $\widehat{\beta}(\mathrm{ep})$ & $\hat{\theta}(\mathrm{ep})$ & $\hat{\lambda}(\mathrm{ep})$ \\
\hline Aquidabã & $8,128(1,021)$ & $5,085(0,560)$ & $3,972(0,482)$ & $1,967(0,181)$ \\
Aracaju & $5,228(0,665)$ & $2,504(0,245)$ & $4,928(0,626)$ & $2,299(0,220)$ \\
Boquim & $5,575(0,703)$ & $2,867(0,288)$ & $4,793(0,596)$ & $2,401(0,232)$ \\
Itabaiana & $4,614(0,576)$ & $2,227(0,211)$ & $4,345(0,563)$ & $1,733(0,154)$ \\
N. S. da Glória & $6,646(0,805)$ & $3,813(0,404)$ & $4,233(0,557)$ & $1,565(0,135)$ \\
Porto da Folha & $8,840(1,096)$ & $6,075(0,682)$ & $4,809(0,640)$ & $1,845(0,167)$ \\
Poço Redondo & $11,710(1,455)$ & $8,754(1,015)$ & $4,479(0,629)$ & $1,359(0,112)$ \\
Umbaúba & $5,161(0,662)$ & $2,385(0,230)$ & $5,177(0,670)$ & $2,306(0,221)$ \\
\hline
\end{tabular}

Utilizando a figura 4 , podem-se comparar os histogramas dos dados observados 
Revista Ciências Exatas e Naturais, Vol.14, n 1, Jan/Jun 2012

com as respectivas fdp ajustadas de $Q$ para as cidades de Aracaju, Boquim, Porto da Folha e Umbaúba.

As estimativas de máxima verossimilhança obtidas também permitem inferir a média de $Q=X /(X+Y)$ através da expressão,

$$
\begin{aligned}
E(Q)= & \frac{B(\alpha+\theta, \beta+\lambda)}{B(\alpha, \beta) B(\beta, \lambda)} \int_{0}^{1} q^{-\theta}(1-q)^{\theta-1} \\
& \times{ }_{2} F_{1}\left(\alpha+\theta, \theta+\lambda ; \alpha+\beta+\theta+\lambda ; 2-\frac{1}{q}\right) d q .
\end{aligned}
$$
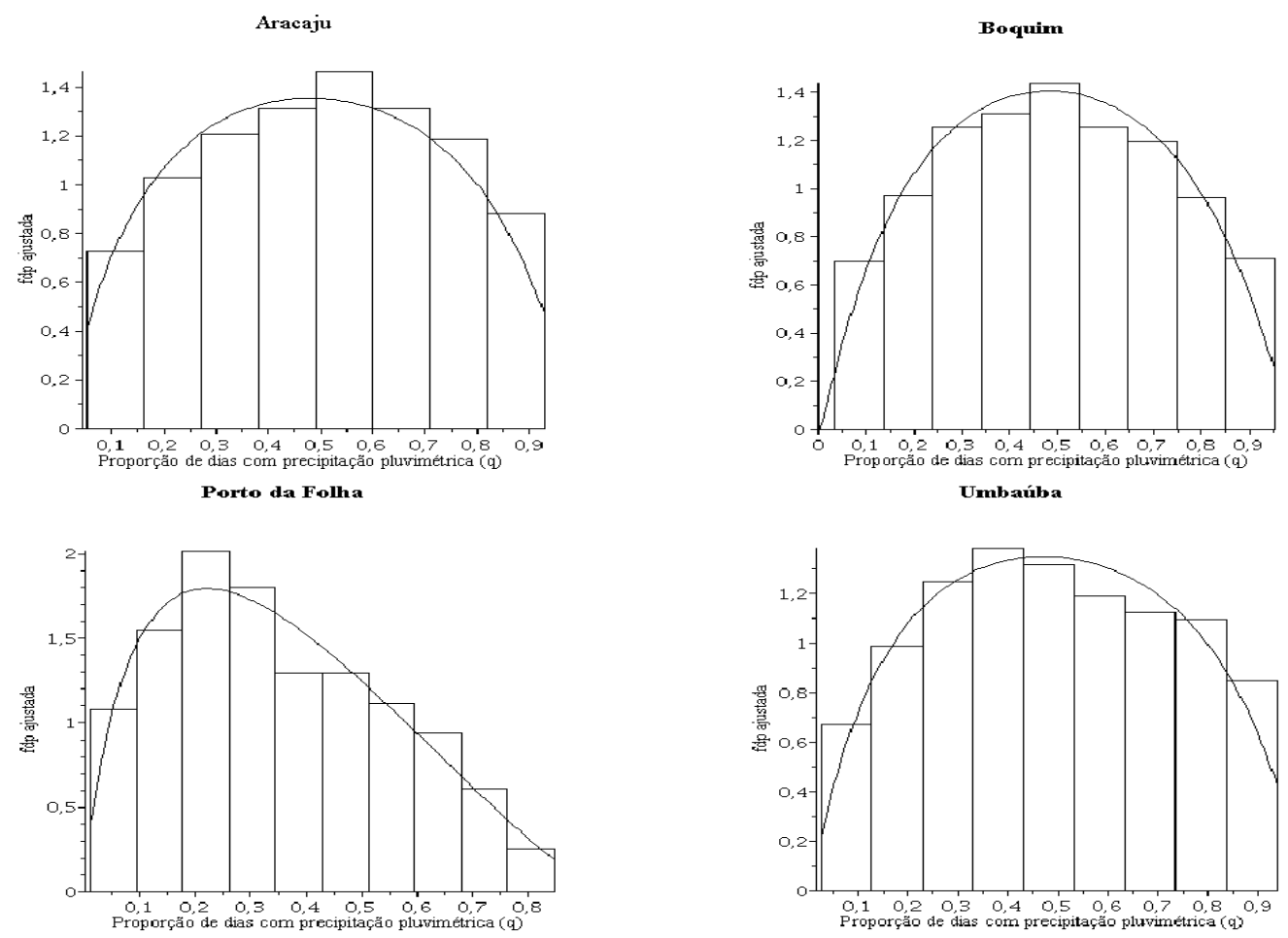

Figura 4. Fdp ajustada de Q para as cidades de Aracaju, Boquim, Porto da Folha e Umbaúba.

Uma forma alternativa de estimar $E(Q)$ é dada pelo Método dos Momentos através da seguinte expressão, 


$$
\widetilde{E}(Q)=\frac{1}{n} \sum_{i=1}^{n} \frac{x_{i}}{x_{i}+y_{i}}
$$

As estimativas oriundas da equação 25] independem de $X$ e $Y$ serem independentes ou beta distribuídas. Os valores das equações (24) e (25) para as oito cidades são exibidos na tabela 4. A proximidade dos valores encontrados é um grande indicativo da validade do modelo.

Tabela 4. Estimativas da média de $Q=X /(X+Y)$

\begin{tabular}{lll}
\hline Cidades & $\mathrm{E}(\mathrm{Q})$ & $\tilde{\mathrm{E}}(\mathrm{Q})$ \\
\hline Aracaju & 0,493 & 0,496 \\
Aquidabã & 0,435 & 0,444 \\
Boquim & 0,491 & 0,492 \\
N. S. da Glória & 0,384 & 0,390 \\
Itabaiana & 0,449 & 0,456 \\
Porto da Folha & 0,363 & 0,366 \\
Poço Redondo & 0,288 & 0,287 \\
Umbaúba & 0,491 & 0,493 \\
\hline
\end{tabular}

A qualidade de ajuste do modelo também foi verificada via gráficos de probabilidade. Para $X$ foi plotado o gráfico de $F_{X}\left(x_{(i)}\right)$ versus $(i-0,375) /(n+0,25)$ como é recomendado por Chambers et al [35], em que $F_{X}(\cdot)$ denota a função de distribuição acumulada de $X$ e $x_{(i)}$ representa os valores amostrais de $X$ em ordem crescente. Da mesma forma para $Y$ foi plotado o gráfico de $F_{Y}\left(y_{(i)}\right)$ versus $(i-0,375) /(n+0,25)$ em que $F_{Y}(\cdot)$ denota a função de distribuição acumulada de $Y$ e $y_{(i)}$ representa os valores amostrais de $Y$ em ordem crescente.

Os gráficos de probabilidade para o ajuste da distribuição de $X$ para quatro cidades são visualizados na figura 5 e a proximidade dos pontos entorno da reta indicam o bom ajuste do modelo. Na figura 6, são exibidos gráficos de probabilidade para o ajuste da distribuição de $Y$ para quatro das oito cidades estudadas, cuja disposição dos pontos próximos à reta indicam o bom ajuste do modelo. 
Revista Ciências Exatas e Naturais, Vol.14, n 1, Jan/Jun 2012
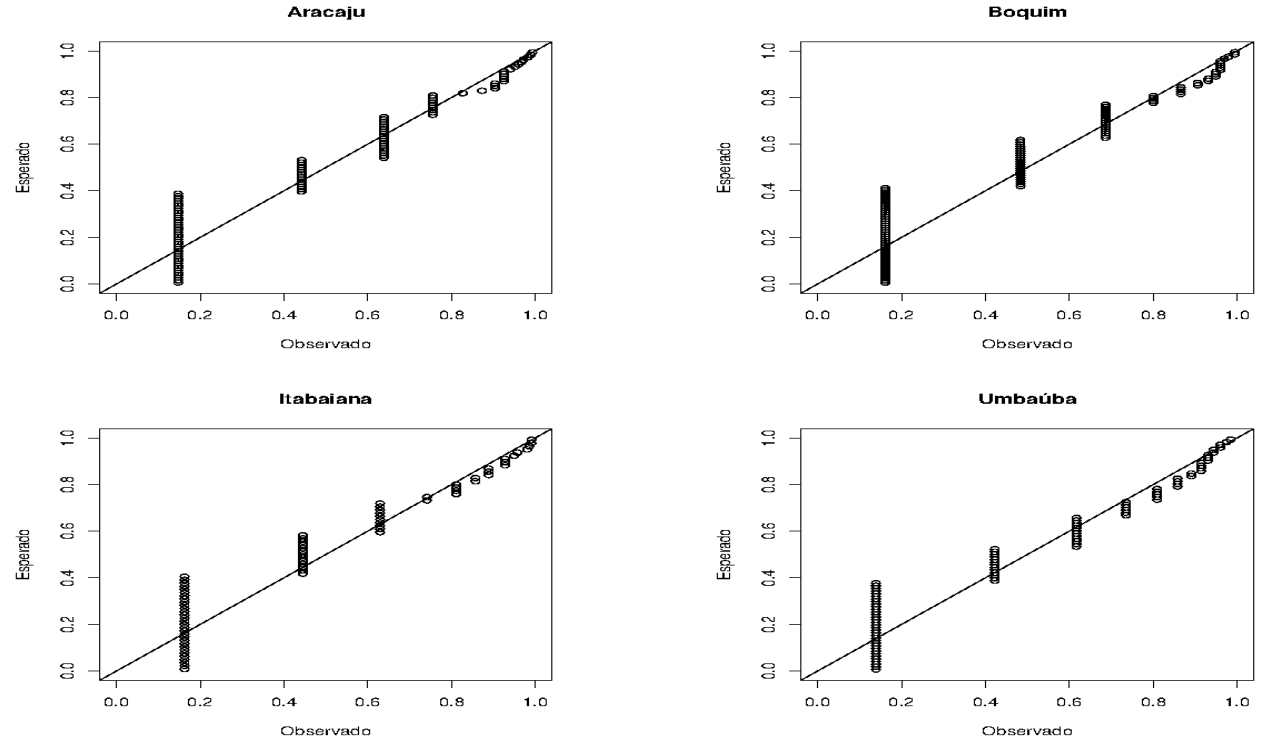

Figura 5. Gráficos de probabilidade para o período de dias com ocorrência de precipitações $(X)$ para quatro cidades selecionadas.
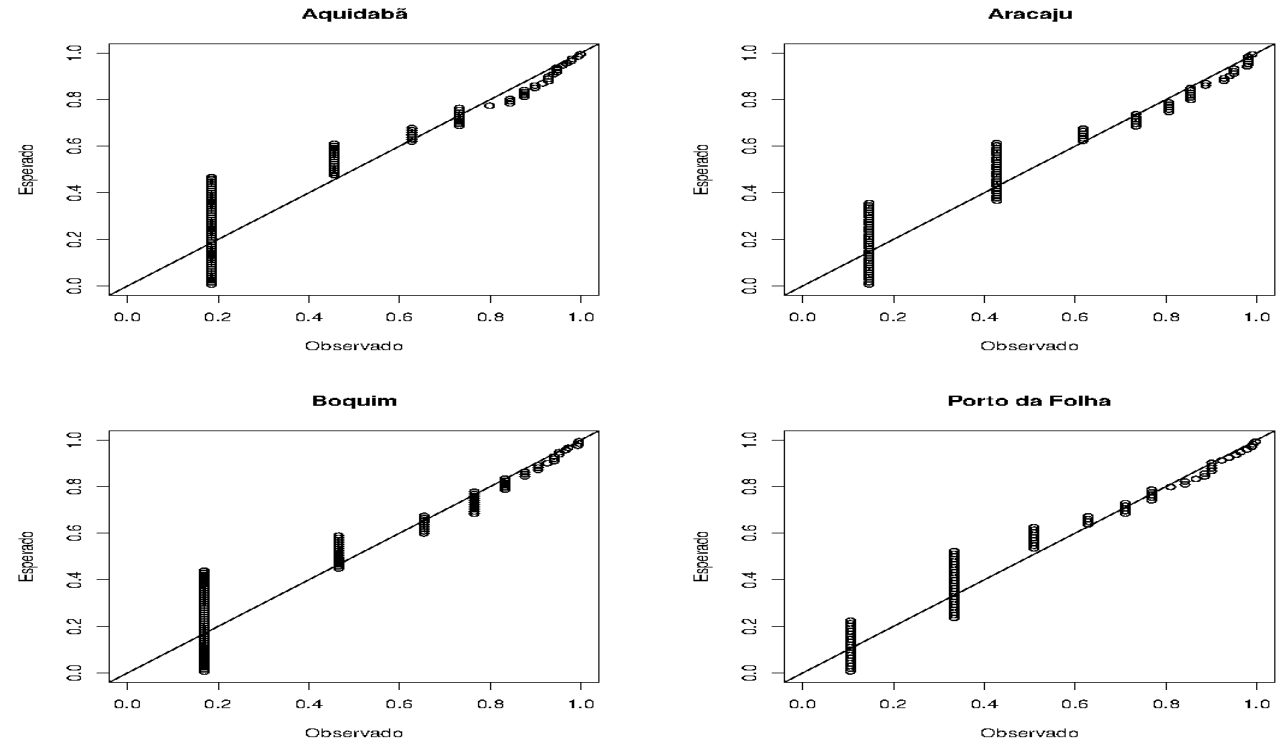

Figura 6. Gráficos de probabilidade para o periodo de dias sem ocorrência de precipitação $(Y)$ para quatro cidades selecionadas. 


\section{Conclusões}

Partindo do fato de $X$ e $Y$ serem beta tipo II distribuídas e independentes, as distribuições das combinações de variáveis $P=X Y, R=X / Y$ e $Q=X /(X+Y)$ apresentaram forma fechada em termos da função hipergeométrica de Gauss. Em particular, a distribuição de $Q$ demonstrou-se bastante flexível, tornando-se uma alternativa interessante em relação à distribuição beta tipo I na modelagem de proporções.

A aplicação do modelo na análise de precipitações pluviométricas ocorridas nas cidades de Aquidabã, Aracaju, Boquim, Nossa Senhora da Glória, Itabaiana, Porto da Folha, Poço Redondo e Umbaúba no Estado de Sergipe apresentou bons resultados, o que foi comprovado pelos P-P plots em função de os pontos em cada gráfico estarem dispostos próximos à reta.

\section{Agradecimentos}

Agradecemos à Coordenadoria para o Aperfeiçoamento de Pessoal de Nível Superior (CAPES), pela bolsa de doutorado do primeiro autor e aos revisores pelas sugestões, as quais contribuíram para o aprimoramento do artigo.

\section{Referências}

[1] SAKAMOTO, H. On the distribution of the product and the quotient of the independent and uniformly distribibuted random variables. Tohoku Math J, v. 49, p. $243-260,1943$.

[2] PODOLSKI, $\mathrm{H}$. The distribution of a product of $\mathrm{n}$ independent random variables with generalized gamma distribution. Demonstr. Math, v. 4, p. 119-123, 1972.

[3] SPRINGER, M. D.; THOMPSON, W. E. The distribution of product beta, gamma and Gaussian random variables. SIAM. J Appl Math, v. 18, p. 721-737, 1970. 
Revista Ciências Exatas e Naturais, Vol.14, n 1, Jan/Jun 2012

[4] STUART, A. Gamma-distributed products of independent random variables. Biometrika, v. 49, p. 564-565, 1962.

[5] HARTER, H. L. On the distribution of Wald's classification statistic. Ann Math Stat, v. 22, p. 58-67, 1951.

[6] WALLGREN, C. M. The distribution of the product of two correlated t variables. J Am Stat Assoc, v. 75, p. 996-1000, 1980.

[7] BHARGAVA, R. P.; KHATRI, C. G. The distribution of product of independent beta random variables with application to multivariate analysis. Ann Inst Stat Math, v. 33, p. 287-296, 1981.

[8] STEECE, B. M. On the exact distribution for the product of two independent beta-distributed random variables. Metron, v. 34, p. 187-190, 1976.

[9] TANG, J.; GUPTA, A. K. On the distribution of the product of independent beta random variables. Stat Probab Lett, v. 2, p. 165-168, 1984.

[10] MALIK, H. I.; TRUDEL, R. Probability density function of the product and quotient of two correlated exponential random variables. Can Math Bull, v. 29, p. 413-418, 1986.

[11] NADARAJAH, S; GUPTA, A. K. On the product and ratio of Bessel random variables. Int J of Mathematics and Mathematical Sciences, v. 18, p. 2977-2989, 2005.

[12] NADARAJAH, S.; ALI, M. M. Pareto variables for hydrological modeling. Water Resour Manag, v. 22, p. 1381-1393, 2008.

[13] ALI, M. M.; PAL, M.; WOO, J. On the ratio of two independet exponentiated Pareto variables. Austrian J Stat, v. 39, p. 329-340, 2010.

[14] KORHONEN, P. J.; NARULA, S. C. The probability distribution of the ratio of the absolute values of two normal variables. J Stat Comput Simul, v. 33, p. 173-182, 1989. 
[15] MARSAGLIA, G. Ratios of normal variables and ratios of sums of uniform variables. J Am Stat Assoc, v. 60, p. 193-294, 1965.

[16] PROVOST, S. B. On the distribution of the ratio of powers of sums of gamma random variables. Pak J Stat, v. 5, p. 157-174, 1989.

[17] BASU, A. P.; LOCHNER, R. H. On distribution of the ratio of two random variables having generalized life distributions. Technometrics, v. 13, p. 281-287, 1971.

[18] PRUDNIKOV, A. P.; BRYCHKOV, Y. A.; MARICHEV,O. I. Integrals and series. Amsterdam: Gordon and Breach, v. 1, p. 808, 1998.

[19] PODOLSKI, $\mathrm{H}$. The distribution of a product of $\mathrm{n}$ independent random variables with generalized gamma distribution. Demonstr Math, v. 4, p. 119-123, 1972.

[20] NADARAJAH, S.; ALI, M. M. On the product and ratio of $\mathrm{t}$ and logistic random variables. Calcutta Stat Ass Bull, v. 55, p. 1-14, 2004.

[21] NADARAJAH, S; KOTZ, S. On the product and ratio of gamma and beta random variables. Allgemeines Stat Archiv, v. 89, p. 435-449, 2005.

[22] NADARAJAH, S. On the product and ratio of Laplace and Bessel random variables. J Appl Math, v. 4, p. 393-402, 2005.

[23] NADARAJAH, S; KOTZ, S. On the product and ratio of Pearson Type VII and Laplace random variables. Austrian J Stat, v. 34, p. 11-23, 2005.

[24] NADARAJAH, S; KOTZ, S. On the product and ratio of gamma and Weibull random variables. Economet Theor, v. 22, p. 338-344, 2006.

[25] NADARAJAH, S; KOTZ, S. On the product and ratio of $\mathrm{t}$ and Bessel random variables. Bull Inst Math Acad Sinica, v. 2, p. 55-66, 2007.

[26] NADARAJAH, S; KOTZ, S. On the p ratio of gamma and Levy random variables. Appl Econ Lett, v. 13, p. 153-157, 2006. 
Revista Ciências Exatas e Naturais, Vol.14, n 1, Jan/Jun 2012

[27] SHAKIL, M.; KIBRIA, B. M. G.; CHANG, K. C. Distributions of the product and ratio of Maxwell and Rayleigh random variables. Stat Papers, v. 49, p. 729-747, 2008.

[28] SPRINGER, M. D.; THOMPSON, W. E. The distribution of product beta, gamma and Gaussian random variables. SIAM. J Appl Math, v. 18, p. 721-737, 1970.

[29] NADARAJAH, S; KOTZ, S. On the linear combination, product and ratio of normal and Laplace random variables. J Franklin Institute, v. 348, p. 810-822, 2011.

[30] JAMBUNATHAN, M. V. Some properties of beta and gamma distributions. The Annals of Math Stat, v. 25, p. 401-405, 1954.

[31] OLDHAM, K.; MYLAND, J.; SPANIER, J. An atlas of functions: with equator, the atlas function calculator. Canadá: Springer Vergland, $2^{a}$ ed., 2009. 700p.

[32] SAKAMOTO, H. On the distribution of the product and the quotient of the independent and uniformly distribibuted random variables. Tohoku Math J, v. 49, p. $243-260,1943$.

[33] BEST, D. J.; ROBERTS, D. E. Algorithm AS 89: The upper tail probabilities of Spearman's rho. Appl Stat, v. 24, p. 377-379, 1975.

[34] SHAKIL, M.; KIBRIA, B. M. G. Exact distribution of the ratio of gamma and Rayleigh random variables. Pak J Stat oper Res, v. 2, p. 87-98, 2006.

[35] CHAMBERS, J.; CLEVELAND, W.; KLEINER, B.; TUKEY, P. Graphical Methods for Data Analisys. Boston: Duxbury Press, p. 395, 1983. 